\title{
Theory of Magnetodynamics Induced by Spin Torque in Perpendicularly Magnetized Thin Films
}

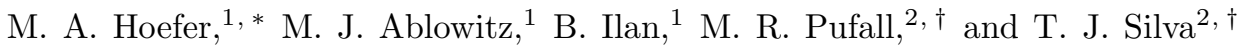 \\ ${ }^{1}$ Department of Applied Mathematics, University of Colorado, Boulder, Colorado 80309-0526, USA \\ ${ }^{2}$ National Institute of Standards and Technology, Boulder, Colorado 80305, USA
}

(Dated: September 12, 2018)

\begin{abstract}
A nonlinear model of spin wave excitation using a point contact in a thin ferromagnetic film is introduced. Large-amplitude magnetic solitary waves are computed, which help explain recent spin-torque experiments. Numerical simulations of the fully nonlinear model predict excitation frequencies in excess of $0.2 \mathrm{THz}$ for contact diameters smaller than $6 \mathrm{~nm}$. Simulations also predict a saturation and red shift of the frequency at currents large enough to invert the magnetization under the point contact. The theory is approximated by a cubic complex Ginzburg-Landau type equation. The mode's nonlinear frequency shift is found by use of perturbation techniques, whose results agree with those of direct numerical simulations.

PACS numbers: 75.30.Ds, 75.40.Gb, 75.70.-i, 76.50.+g
\end{abstract}

Generation of spin waves at microwave frequencies due to spin-momentum transfer (SMT) in a thin magnetic multilayer is paving the way to a new frontier in magnetodynamics. Initial predictions, in the small amplitude limit, made by Slonczewski [1] were recently validated experimentally 2, 3, 4]. In refs [2, 4], microwaves were generated by the application of a dc current through a nanocontact into a trilayer of alternating ferromagnetic and nonmagnetic materials (see fig 1). Several models [5, 6, 7] have been suggested to explain this phenomenon. More recent work has neglected spatial variation [8, 9, 10]. In this study, a theory of nonlinear spatially nonuniform magnetodynamics is developed and analyzed. Microwave emission from point contacts has potential applications in communications and fast, highdensity magnetic data storage.

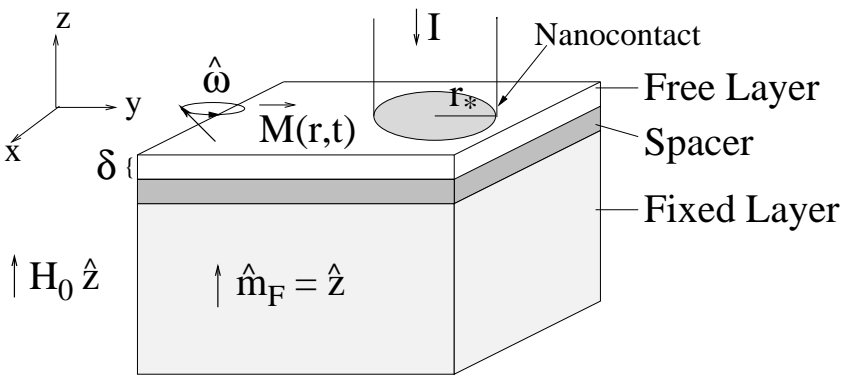

FIG. 1: Magnetic multilayer with magnetization $\vec{M}$ precessing in the free layer with frequency $\hat{\omega}$. The magnetization of the lower ferromagnetic layer is fixed with orientation $\hat{m}_{F}$. A nonmagnetic spacer is sandwiched between two ferromagnetic layers. Typical parameters are: $r_{*}=20 \mathrm{~nm}$ and $\delta=5 \mathrm{~nm}$.

The system depicted in fig. 1 has been shown experimentally to give rise to high-frequency microwaves inferred to be caused by steady-state precession of magnetization in the ferromagnetic free layer $\left(\mathrm{Ni}_{80} \mathrm{Fe}_{20}\right)$ [4]. An external magnetic field $\vec{H}_{0}$ is oriented perpendicular to the film plane. When a dc current, $I$, is applied through a point contact into the multilayer $\mathrm{Co}_{90} \mathrm{Fe}_{10} / \mathrm{Cu} / \mathrm{Ni}_{80} \mathrm{Fe}_{20}$, the conduction electrons' spins induce a torque on the magnetization in the free layer due to conservation of angular momentum [1, 5]. This SMT torque opposes the intrinsic damping of the free layer and produces complex dynamics. Slonczewski's linear theory of spin wave excitation for point contacts on magnetic multilayers predicts a threshold current for the excitation of steady precession of magnetization 11]. However, it does not accurately predict the high frequencies seen in large amplitude experiments nor the dependence of the frequency on current.

Understanding the SMT effect in point contacts with a perpendicular geometry is of great interest as it is a microwave source that yields the narrowest line widths and highest output powers [4, 12] when compared to other SMT geometries, such as nanopillars [3] and inplane fields [13]. In addition, this configuration is most amenable to analytical study given the high symmetry of the problem. Insights derived from this simpler geometry may then be employed in solving the more advanced problems of in-plane or oblique angled fields.

In this Letter, beginning with a nonlinear vectorial equation, we derive a new complex cubic GinzburgLandau (CGL) equation for weakly nonlinear excitations. The dipole coupling and exchange nonlinearities are shown to be responsible for the higher frequencies. Steady-state precessing modes for the nonlinear vectorial model are found via numerical integration. The calculated frequencies lie in the range of experimental observations [4]. Using this model, much higher frequencies $(0.2 \mathrm{THz})$ are predicted for systems with contact radii on the order of $r_{*}=3 \mathrm{~nm}$. A surprising prediction is the saturation and red shift of frequency for currents large enough to invert the magnetization under the point contact. This is a direct consequence of the exchange term included in our model.

The magnetization $\vec{M}=\left(M_{x}, M_{y}, M_{z}\right)$ in the free layer 
can be described by the vectorial equation

$$
\begin{aligned}
\frac{\partial \vec{M}}{\partial \tau}= & \underbrace{-|\gamma| \mu_{0} \vec{M} \times \vec{H}_{\mathrm{eff}}}_{\text {precession }}-\underbrace{\frac{2}{M_{s}^{2} T_{2}} \vec{M} \times\left(\vec{M} \times \vec{H}_{\mathrm{eff}}\right)}_{\text {Landau-Lifshitz damping }}+ \\
& \underbrace{\beta(\vec{x}) \vec{M} \times\left(\vec{M} \times \hat{m}_{\mathrm{F}}\right)}_{\text {Slonczewski SMT torque }},
\end{aligned}
$$

where the precessional and damping terms are driven by the effective magnetic field $\vec{H}_{\text {eff }}=H_{0} \hat{z}-M_{z} \hat{z}+$ $\frac{D}{|\gamma| \mu_{0} M_{s} \hbar} \nabla^{2} \vec{M}$. This field consists of the applied magnetic field, $H_{0} \hat{z}$, the demagnetizing field due to axial dipole coupling, $-M_{z} \hat{z}$, and the exchange field proportional to $\nabla^{2} \vec{M}$ [14]. The Landau-Lifshitz form of damping is a commonly used phenomenological term that drives the magnetization to align with the total field. The Slonczewski SMT torque term is derived in [1] and assumes a large, bulk ferromagnetic layer with fixed magnetization direction $\hat{z}$. Relevant parameters are the gyromagnetic ratio $\left(\gamma=g \mu_{B} / \hbar\right)$, the spectroscopic splitting factor $(g)$, the Bohr magneton $\left(\mu_{B}\right)$, Planck's constant divided by $2 \pi(\hbar)$, the free space permeability $\left(\mu_{0}\right)$, the exchange coupling parameter $(D)$, the magnetization volume density at saturation $\left(M_{s}\right)$, and the transverse relaxation time $\left(T_{2}\right) ; \beta(\vec{x})$ is a driving term depending on the current discussed below.

We assume zero temperature and negligible crystalline anisotropy in order to gain insight into the basic mode structure facilitated by the fundamental physics associated with SMT in point contacts. Such effects can be accounted for by modification of eq (1) to include a random fluctuation term and an effective anisotropy field. Also, since the thickness of the free layer is small, $\delta \approx 5$ $\mathrm{nm}$, the in-plane components of the dipole field contribution to $\vec{H}_{\text {eff }}$ can be neglected [15].

The magnetization is assumed to have rotational symmetry: the spatial variation of the magnetization depends solely on the distance $r$ from the center of the point contact. Then, the current term, $\beta$, is given by

$$
\beta(r)=\frac{I \hbar \epsilon \gamma}{2 M_{s}^{2} \delta \pi r_{*}^{2} e} \Phi\left(r_{*}-r\right), \Phi\left(r_{*}-r\right)=\left\{\begin{array}{ll}
1 & r \leq r_{*} \\
0 & r>r_{*}
\end{array},\right.
$$

where $\epsilon$ is the SMT efficiency, $\delta$ is the thickness of the free layer, $e$ is the charge of an electron, $r=\left(x^{2}+y^{2}\right)^{1 / 2}$, and $\Phi\left(r_{*}-r\right)$ is the Heaviside step function defining the point contact to be a circle of radius $r_{*}$. The efficiency $\epsilon$ is in principle a complicated function of many parameters, including (but not limited to) microscopic details of the interfaces in question [6, 11]. Many of the approximations used in the calculation of $\epsilon$ ignore the lateral geometry of the actual experiments, choosing instead to use a more tractable 1D calculation. Since $\epsilon$ is difficult to approximate, let alone calculate, and is not the subject of this paper, we will treat $\epsilon$ as a constant fitting parameter.
By taking the dot product of equation (1) with $\vec{M}$, one sees that the magnetization of the free layer is locally conserved for all times, i.e., $|\vec{M}|=M_{s}=$ constant, or

$$
M_{s}^{2}=M_{x}^{2}(r, \tau)+M_{y}^{2}(r, \tau)+M_{z}^{2}(r, \tau), r \geq 0, \tau \geq 0 .
$$

Consider the standard normalization, $\vec{m}=\vec{M} / M_{s}=$ $\left(m_{x}, m_{y}, m_{z}\right), t=\omega_{M} \tau, \rho=r / l_{\text {ex }}$, where $\omega_{M} \equiv \gamma \mu_{0} M_{s}$ and the exchange length is $l_{\mathrm{ex}} \equiv \sqrt{D / \gamma \mu_{0} M_{s} \hbar}$. For the analysis, it is convenient to encode the transverse components of $\vec{m}, \vec{m}_{\perp}=\left(m_{x}, m_{y}\right)$, in the complex quantity $m=m_{x}+i m_{y}$. The axial component of the magnetization is treated as a perturbation from the equilibrium solution $\vec{m} \equiv \hat{z}$, so $m_{z}=1-f$, where $f$ is the normalized perturbation. Evaluating the vector cross products in (10) leads to

$$
\begin{aligned}
i \frac{\partial m}{\partial t}= & m \nabla^{2} f+(1-f+i \alpha) \nabla^{2} m+(1-f-h) m \\
& +i\left\{j \Phi\left(\rho_{*}-\rho\right)-\alpha(h-(1-f))\right\}(1-f) m \\
& +i \alpha\left\{|\nabla f|^{2}+|\nabla m|^{2}\right\} m \\
\frac{\partial f}{\partial t}= & \operatorname{Im}\left(m^{*} \nabla^{2} m\right)+\left\{j \Phi\left(\rho_{*}-\rho\right)-\alpha(h-(1-f))\right\}|m|^{2} \\
& +\alpha\left\{\nabla^{2} f-\left(|\nabla m|^{2}+|\nabla f|^{2}\right)(1-f)\right\},
\end{aligned}
$$

where $\nabla^{2} \equiv \frac{\partial^{2}}{\partial \rho^{2}}+\frac{1}{\rho} \frac{\partial}{\partial \rho}$. The dimensionless parameters for applied field, damping, and current are respectively

$$
h=\frac{H_{0}}{M_{s}}, \alpha=\frac{2}{\gamma \mu_{0} M_{s} T_{2}}, j=\frac{\hbar \epsilon}{2 M_{s}^{2} e \mu_{0} \pi r_{*}^{2} \delta} I .
$$

The coupled system (3) is equivalent to the model (11). Since the patterned multilayer mesa used in experiments is two orders of magnitude wider than the point contact [4], we assume that the multilayer has infinite extent in the $x y$ plane, hence $0 \leq \rho<\infty$.

The nominal parameter values used in this paper are $r_{*}=20 \mathrm{~nm}, \epsilon=0.26, D=4 \mathrm{meV} \cdot \mathrm{nm}^{2}, M_{s}=640 \mathrm{kA} / \mathrm{m}$, $\delta=5 \mathrm{~nm}, \gamma=1.85 \cdot 10^{11} \mathrm{~Hz} / \mathrm{T}, \omega_{M}=23.68 \mathrm{GHz}, l_{\mathrm{ex}}=6.40$ $\mathrm{nm}$, and $\alpha=0.0112$. We show that by using only $\epsilon$ as a fitting parameter, the theoretical data match experiment well. Except where noted, the parameter values $h=1.1$ and $\rho_{*}=3.12$ are used.

In order to develop a perturbation scheme, we introduce a small parameter, $\frac{a^{2}}{2} \ll 1$, and a rescaling of equation (3), $m=a \tilde{m}$. The parameter $a$ represents the magnitude scale of the transverse magnetization at the center of the point contact. Using the approximation, $f \approx \frac{a^{2}}{2}|\tilde{m}|^{2}$ for the saturation condition, substituting this approximation into eq (3), and keeping only first order terms in 
$a^{2}$, we find that $\tilde{m}$ satisfies a CGL-type equation,

$$
\begin{aligned}
i \frac{\partial \tilde{m}}{\partial t}= & (1+i \alpha) \nabla^{2} \tilde{m}-(h-1) \tilde{m}+i\{j \Phi-\alpha(h-1)\} \tilde{m} \\
& +\frac{a^{2}}{2}\{\underbrace{-|\tilde{m}|^{2} \tilde{m}}_{\text {dipole coupling }}+i[j \Phi-\alpha(h-2)]|\tilde{m}|^{2} \tilde{m} \\
& +\underbrace{\left[\tilde{m} \nabla^{2} \tilde{m}^{*}+2(1+i \alpha)|\nabla \tilde{m}|^{2}\right] \tilde{m}}_{\text {nonlinear exchange }}\} .
\end{aligned}
$$

Seeking a steady-state solution of the form $\tilde{m}(\rho, t)=$ $e^{i \hat{\omega} t} \phi(\rho), \hat{\omega}=h-1-\omega$ yields a nonlinear eigenvalue problem with boundary conditions $\phi(0)=1, d \phi / d \rho(0)=0$, $\lim _{\rho \rightarrow \infty} \phi(\rho)=0$. The corresponding linear eigenvalue problem was solved by Slonczewski [11] with two real eigenvalues $\omega$ and $j$. This suggests the asymptotic expansions

$$
\begin{gathered}
\phi(\rho)=\phi^{(0)}(\rho)+\frac{a^{2}}{2} \phi^{(1)}(\rho)+\cdots \\
\omega=\omega^{(0)}+\frac{a^{2}}{2} \omega^{(1)}+\cdots, \quad j=j^{(0)}+\frac{a^{2}}{2} j^{(1)}+\cdots .
\end{gathered}
$$

Slonczewski's linear result is summarized below.

Linearized solution [11]: The leading order mode $\phi^{(0)}(\rho)$ in the expansion (5) is

$\phi^{(0)}(\rho)=\left\{\begin{array}{lc}J_{0}\left(k_{i} \rho\right) & 0 \leq \rho \leq \rho_{*} \\ c H_{0}^{(2)}\left(k_{o} \rho\right) & \rho_{*}<\rho\end{array}, \quad c=\frac{J_{0}\left(k_{i} \rho_{*}\right)}{H_{0}^{(2)}\left(k_{o} \rho_{*}\right)}\right.$,

$k_{i}=\left(\frac{-\omega^{(0)}+i\left(j^{(0)}-\alpha(h-1)\right)}{1+i \alpha}\right)^{1 / 2}, k_{o}=\left(\frac{-\omega^{(0)}-i \alpha(h-1)}{1+i \alpha}\right)^{1 / 2}$, where the linear eigenvalues $\omega^{(0)}$ and $j^{(0)}$ are determined by the real and imaginary parts of

$$
k_{i} H_{0}^{(2)}\left(k_{o} \rho_{*}\right) J_{1}\left(k_{i} \rho_{*}\right)=k_{o} H_{1}^{(2)}\left(k_{o} \rho_{*}\right) J_{0}\left(k_{i} \rho_{*}\right) .
$$

Equation (6) is solved numerically using a nonlinear root finder. A doubly-discrete set of eigenvalues for frequency and current is found. We consider only the lowest eigenvalues $\omega^{(0)}$ and $j^{(0)}$, where $j^{(0)}$ is the critical current for linear spin wave excitations [1]].

The predicted frequency of the ground state mode, $\hat{\omega} \sim h-1-\omega^{(0)}$, is valid only for very small amplitudes $a$, whereas experiments produce much higher frequencies [4]. The nonlinearity in (4) gives rise to a frequency shift which, through the use of the Poincaré method [16], helps explain this disparity.

Nonlinear solution: The first order nonlinear frequency and critical current shifts $\omega^{(1)}$ and $j^{(1)}$ are determined by the linear system

$$
\left[\begin{array}{cc}
\operatorname{Re}\left(I_{2}\right) & \operatorname{Im}\left(I_{1}\right) \\
\operatorname{Im}\left(I_{2}\right) & -\operatorname{Re}\left(I_{1}\right)
\end{array}\right]\left[\begin{array}{c}
\omega^{(1)} \\
j^{(1)}
\end{array}\right]=\left[\begin{array}{c}
\operatorname{Re}\left(I_{3}\right)+\operatorname{Im}\left(I_{4}\right) \\
\operatorname{Im}\left(I_{3}\right)-\operatorname{Re}\left(I_{4}\right)
\end{array}\right]
$$

where $I_{k}$ depend only on the leading-order solution:

$$
\begin{aligned}
I_{1} \equiv & \frac{\rho_{*}^{2}}{2}\left\{J_{0}^{2}\left(k_{i} \rho_{*}\right)+J_{1}^{2}\left(k_{i} \rho_{*}\right)\right\} \\
I_{2} \equiv & I_{1}-\frac{c^{2} \rho_{*}^{2}}{2}\left\{\left(H_{0}^{(2)}\left(k_{o} \rho_{*}\right)\right)^{2}+\left(H_{1}^{(2)}\left(k_{o} \rho_{*}\right)\right)^{2}\right\} \\
I_{3} \equiv & \int_{0}^{\infty}\left\{-\left|\phi^{(0)}\right|^{2}+\phi^{(0)} \nabla^{2} \phi^{(0) *}+2\left|\nabla \phi^{(0)}\right|^{2}\right\} \phi^{(0)^{2}} \rho \mathrm{d} \rho \\
I_{4} \equiv & \int_{0}^{\infty}\left\{\alpha(2-h)\left|\phi^{(0)}\right|^{2}-2 \alpha\left|\nabla \phi^{(0)}\right|^{2}\right\} \phi^{(0)^{2}} \rho \mathrm{d} \rho \\
& +j^{(0)} \int_{0}^{\rho_{*}}\left|\phi^{(0)}\right|^{2} \phi^{(0)^{2}} \rho \mathrm{d} \rho .
\end{aligned}
$$

The above perturbation method provides a means for calculating the nonlinear frequency and current shifts, $\omega^{(1)}$ and $j^{(1)}$, of a mode solution to (4) and helps explain experimental results 2]. The frequency and critical current have a parabolic dependence on the transverse amplitude $a$

$$
\hat{\omega} \sim h-1-\omega^{(0)}-\frac{a^{2}}{2} \omega^{(1)}, \quad j \sim j^{(0)}+\frac{a^{2}}{2} j^{(1)} .
$$

We note that using equations (77), the relation between $\hat{\omega}$ and $j$ is linear because both scale as $a^{2}$.

The first-order nonlinear contributions from equation (4) due to exchange and dipole coupling were compared. Using equations (7), the frequency and current dependence as parameterized by $a$ were calculated when the exchange and dipole coupling nonlinearities were removed from the calculation. We find that if nonlinear exchange terms are ignored, the relative error in the slope $d \hat{\omega} / d j$ is approximately $10 \%$ for $r_{*}=20 \mathrm{~nm}$, whereas for $r_{*}<12$ $\mathrm{nm}$ the relative error is greater than $25 \%$.

Experiments [4] show that for large currents, and hence larger amplitudes, the frequency/current relationship significantly deviates from linearity. In order to account for this, equation (31) was solved numerically using the projection method where one renormalizes the magnetization after each time step in order to preserve the local conservation of $\vec{M}$ (2) (see [17]). The steady states of this parametrically forced/damped system are attractors. The initial condition $m(\rho, 0)=a \phi^{(0)}(\rho)$ was evolved in time over a large domain $(0 \leq \rho \leq 100)$ until the solution relaxed to a steady state. The mode frequency was numerically recovered from the phase of the precessing transverse component as $\hat{\omega}=\frac{d}{d t} \arg [m(0, t)], t \rightarrow \infty$.

Typical modes, their frequencies, and currents are shown in fig. 2 The transverse mode envelope $\pm|m|=$ $\pm\left(m_{x}^{2}+m_{y}^{2}\right)^{1 / 2}$ and the $y$-component of magnetization $m_{y}$ show slow decay, which means that spin waves are continually radiating away from the point contact, and hence energy from the contact region is lost into the surrounding medium.

The plot of frequency versus current in fig. [3] shows a comparison between numerical simulation of (3), perturbation theory (7]), and experiment [4]. For current excitations larger than $j_{\max }$, the solitary-wave mode structure 


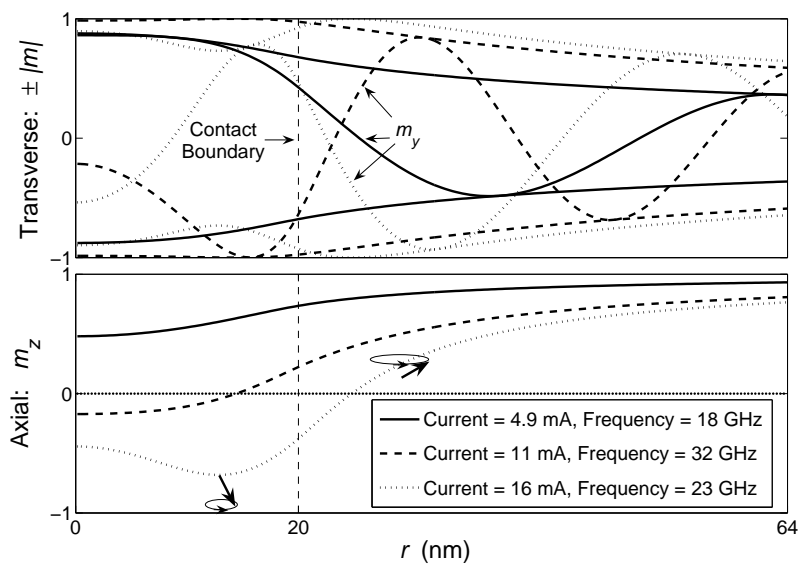

FIG. 2: Spatial dependence of fully nonlinear modes with excitation current and frequency. Top: transverse envelopes $\pm|m|= \pm \sqrt{m_{x}^{2}+m_{y}^{2}}$ and associated spin waves $m_{y}$. Bottom: axial components $m_{z}$. For $j \geq j_{\max }$, the transverse mode magnitude is no longer monotonically decreasing since the axial component of magnetization is negative $\left(m_{z}<0\right)$ in the point contact. Frequency and current values for each mode are shown as circles in fig. 3

becomes highly nonlinear (compare with fig. 2). There is a maximum frequency and corresponding current for a given physical system $\left(j_{\max }, \hat{\omega}_{\max }\right)$. As the dashed mode in fig. 2] shows, frequency saturation corresponds to magnetization precession along the equator near the center of the point contact $\left(m_{z} \approx 0\right)$. For currents less than $j_{\max }$, the transverse mode magnitude has a monotonically decreasing dependence on $r$. For larger currents, the mode is not monotonic and develops interesting structure inside the point contact.

Fig. 3 shows that, as the point contact size is increased, the slope of frequency versus current decreases because the slope depends on SMT torque (inversely proportional to $r_{*}^{2}$ ) and damping due to spin wave generation (inversely proportional to $r_{*}$ in the linear approximation 11]). The comparison of the simulated results $(*)$ with experiment is remarkable when the SMT efficiency is taken to be $\epsilon=0.8$. As the experimental values of the frequency level out, full saturation is being reached. This is the first model to qualitatively capture this type of behavior.

Nominal values for $\epsilon$ are on the order of 0.25 for most ferromagnetic metals 18. The fact that $\epsilon>0.25$ is used to fit the experimental data suggests that further refinement of the model is desirable.

The dependence of frequency on applied magnetic field $H_{0}$ is depicted in fig. 4 With the fitted SMT efficiency parameter, the theory compares well with experiment. The mode structure is highly nonlinear because the transverse amplitude is $|m(0, t)| \approx 0.98$, which precludes the use of eq (7). However, a full numerical solution is required for only one particular value of $h$. Since $d \hat{\omega} / d h \approx$ constant for $\alpha \ll 1$, once the parameters $\alpha, j$, and $\rho_{*}$

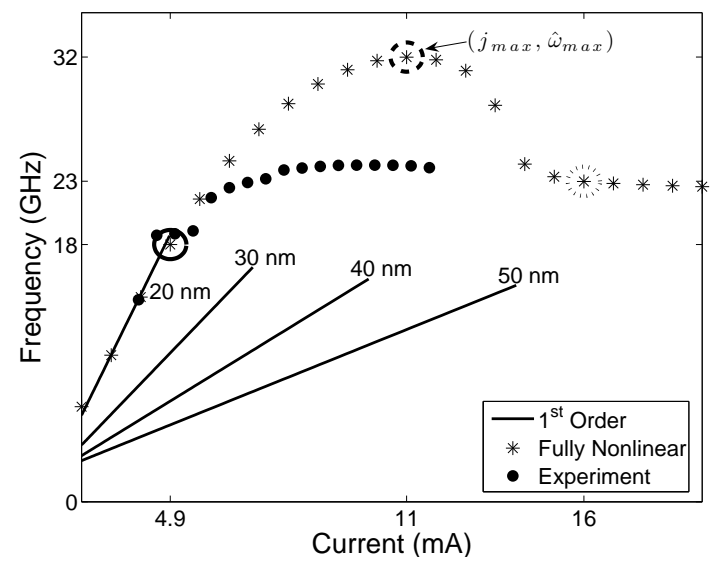

FIG. 3: Precession frequency as a function of current with comparison between fully nonlinear modes (direct numerical simulation of eq (3), the perturbation result (7), and experiments conducted using $r_{*}=20 \mathrm{~nm}$ point contacts [4]. Large circles represent the frequency and current of modes plotted in fig. [2] The straight lines were generated by perturbation theory (eq (7) ) for the contact radii denoted. Note the excellent agreement between numerical simulation, perturbation theory, and experiment for small currents. The parameters have nominal values except $\epsilon=0.8$.

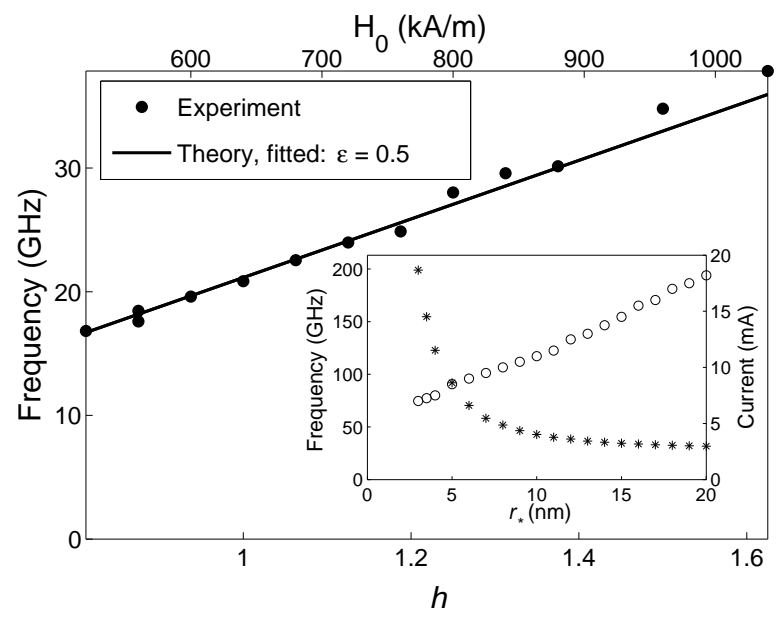

FIG. 4: Frequency as a function of applied field $h$. Comparison between fully nonlinear modes (direct numerical simulation of eq (3) ) and experiments [4]. Parameters are $I=10$ $\mathrm{mA}$ and fitted $\epsilon=0.5$. Inset: maximum frequency $(*)$ and current (o) versus contact radius.

are chosen, the y-intercept for the approximate linear relationship between $\hat{\omega}$ and $h$ is fixed. The slope of the line does not change. The theory predicts the slope very accurately. In fig. 4 inset, much higher frequencies are predicted by shrinking the size of the point contact. Using the method stated above, we predict that frequencies in the $0.2 \mathrm{THz}$ range are attainable for a point contact with a radius of $3 \mathrm{~nm}$. We find that as the point contact is made smaller, the current decreases linearly for the same reasons stated earlier in regard to the $\hat{\omega}(j)$ slope, 
while the frequency increases in proportion to $1 / r_{*}^{2}$ due to exchange-mode spin-wave dispersion.

In summary, a new model of magnetic excitations in point contact structures that includes nonlinearities due to both exchange and dipole coupling is introduced. Spatially dependent steady-state modes are calculated. This represents a large step forward in the explanation of the phenomena observed in recent experiments.

This work was supported by NSF grants DMS0303756, VIGRE DMS-9810751, AFOSR grant F-462003-0250, and the DARPA SPinS program.

* Electronic address: hoefer@colorado.edu

† Contribution of the U.S. Government. Not subject to copyright.

[1] J. C. Slonczewski, J Magn Magn Mater 159, L1 (1996).

[2] M. Tsoi et al., Phys Rev Lett 80, 4281 (1998).

[3] S. I. Kiselev et al., Nature 425, 380 (2003).

[4] W. H. Rippard et al., Phys Rev Lett 92, 027201 (2004).
[5] L. Berger, Phys Rev B 54, 9353 (1996).

[6] M. D. Stiles and A. Zangwill, Phys Rev B 66, 014407 (2002).

[7] Y. B. Bazaliy, B. A. Jones, and S.-C. Zhang, Phys Rev B 57, R3213 (1998).

[8] S. M. Rezende, F. M. de Aguiar, and A. Azevedo, Phys Rev Lett 94, 037202 (2005).

[9] G. Bertotti et al., Phys Rev Lett 94, 127206 (2005).

[10] A. N. Slavin and P. Kabos, IEEE T Magn 41, 1264 (2005).

[11] J. C. Slonczewski, J Magn Magn Mater 195, L261 (1999).

[12] W. H. Rippard et al., Phys Rev Lett 95, 067203 (2005).

[13] W. H. Rippard et al., Phys Rev B 70, 100406(R) (2004).

[14] L. D. Landau and E. M. Lifshitz, Statistical Physics Part 2 (Butterworth-Heinemann, 1980), 3rd ed.

[15] M. L. Schneider, A. B. Kos, and T. J. Silva, Appl Phys Lett 85, 254 (2004).

[16] J. D. Cole, Perturbation Methods in Applied Mathematics (Blaisdell, Waltham, MA, 1968).

[17] W. E and X.-P. Wang, SIAM J Numer Anal 38, 1647 (2000).

[18] M. R. Pufall, W. H. Rippard, and T. J. Silva, Appl Phys Lett 83, 323 (2003). 